\title{
Analysis and Compensation of Chromatic Dispersion in Long-hauls Optical Coherent System
}

\author{
Narimane Hadjadji ${ }^{*}$, Rachid Hamdi \\ 1 Telecommunications Laboratory (LT), Faculty of Science and Technology, University of May 8, 1945 Guelma, 24000 Guelma, \\ P. O. B. 401, Algeria \\ * Corresponding author, e-mail: hadjadji.narimane@univ-guelma.dz
}

Received: 29 October 2019, Accepted: 13 July 2020, Published online: 16 September 2021

\begin{abstract}
In this paper, we demonstrate the efficiency of Electronic Dispersion Compensation (EDC) for coherent optical systems based on Polarization Division Multiplexed Quadrature Phase Shift Keying (PDM-QPSK). The performance of the proposed system is tested using a pulse that has been recently used in the presence of nonlinear effects.

The proposed system is compared to the 0.3RZ-PDM-QPSK system at the optimum launched power under different symbol-rates and lengths of transmission. The simulation results confirm that the proposed method enhances the system performance. In addition, it secures a low penalty that is below $0.6 \mathrm{~dB}$. As a result, the feasible transmission distance is improved by $29 \%, 20.15 \%$, and $26.7 \%$, at 14 GBaud, 28 Gbaud, and 56 Gbaud, respectively.
\end{abstract}

\section{Keywords}

coherent optical systems, chromatic dispersion compensation, dual polarisation quadrature phase shift keying modulation format, Digital Signal Processing (DSP), Bit Error Rate (BER)

\section{Introduction}

High capacity ultra-long-distance transmission is a major concern in coherent optical systems with the least possible equipment and power consumption. Multi-level modulation format together with Polarization Division Multiplexing is the solution for high Spectral Efficiency (SE). PDM-QPSK has become the standard Long-Haul transmission modulation format for backbone applications. Therefore, it allows for transmitting the signal that is encoded at both phase and polarization [1-3].

Additionally, the coherent receivers with algorithms of Digital Signal Processing (DSP) enhance the mitigation of channel impairments [4]. The Chromatic Dispersion (CD) becomes the main limitation for the single long-haul coherent optical PDM-QPSK systems. The signal is distorted by the temporal broadening of the pulses due to propagation at different velocities during the optical link [5].

The first study was carried out using Dispersion Compensating Fiber (DCF) in the optical domain. On the other hand, higher Amplified Simultaneous Emission (ASE) noise was being generated by the optical amplifier. It has led to a lower Optical Signal to Noise Ratio (OSNR) and increasing the complexity and cost of the system [6].
As a solution, the Electronic Dispersion Compensation (EDC) has been performed through DSP in the time and frequency domain. Time Domain Equalizers (TDE) are used only in short distances because of their higher power consumption and computational complexity. They are replaced by the Frequency Domain Equalizer (FDE) because of its long reach transmission with lower computational complexity [7-13].

Moreover, other techniques have been suggested, such as choosing the optimum pulse shape for the CD tolerance by reducing the spectral occupancy and minimizing intersymbol interference [14]. The RZ-QPSK modulation format has a wide spectrum used together with the narrow optical filter to decrease the intersymbol interference. In addition, the RZ-QPSK higher peak power can enhance receiver sensitivity. Then, it minimizes the OSNR penalty, which is required in long-distance transmission systems $[15,16]$.

In 2014, Jain and Kumar [17] proposed a new pulse shape that can improve the performance of a QPSK, PM-QPSK and 16-star QAM systems for 25 GBaud symbol rate. It is compared to the rectangular pulse under OSNR of 
$6 \mathrm{~dB}$ in terms of the effects of laser Phase Noise (PN), Chromatic Dispersion, and Self-Phase Modulation (SPM).

In 2017, Xu et al. [18] investigated a different Chromatic Dispersion compensation for 28-Gbaud dual-polarization QPSK over $2000 \mathrm{~km}$ Single Mode Fiber (SMF). The DCF is applied as optical compensation. The methods were studied for EDC. The Time-Domain Equalization (TDE), the Frequency-Domain Equalization (FDE) and the Least Mean Square (LMS) adaptive equalization. It concluded that electronic compensation is recommended for long-reach optical systems in the presence of laser Phase Noise (PN).

Increasing the symbol rate reduces the maximum achievable distance of the single transmission under the influence of the Chromatic Dispersion and SPM effects.

The novelty of this research is to implement the pulse suggested by Jain and Kumar [17] to deal with the intersymbol-interference (ISI) and improve the tolerance of Chromatic Dispersion in the long-haul PDM-QPSK systems in presence of Self-Phase Modulation effect for symbol rate up to 56 Gbaud. In addition, applying for electronic compensation in the digital domain decreases the optical complexity. This allows improvements in the optimal reachable transmission while it increases the symbol rate compared to the reference system.

The performance of the proposed system is evaluated and compared with the 0.3RZ-PDM-QPSK system. The Frequency Domain Equalizer is chosen as a technique for Chromatic Dispersion compensation after proving its efficiency. Furthermore, we have discussed the simulation results of the two systems performances in terms of Bit Error Rate (BER), Q factor and OSNR penalty. It is found that the single carrier $112 \mathrm{Gbps}$ proposed system can be transmitted over $6412.5 \mathrm{~km}$ of Standard Single-Mode Fiber (SSMF). The transmission penalties are reduced by up to $0.5 \mathrm{~dB}$. In addition, we have presented the transmission results of both systems including multiple symbol rates (14, 28, 56 Gbaud, respectively) over different

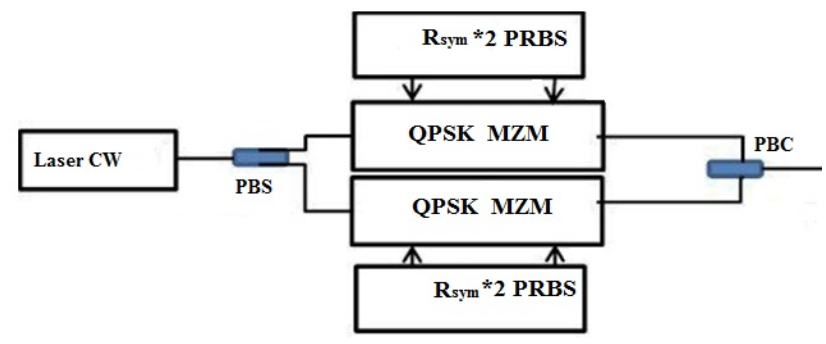

(a) transmission distances. It is shown that the suggested system gives better BER performance and smaller OSNR penalty with extended optical transmission reach.

This paper is organized as follows. Section 2 describes the simulation setup. Also, the two dispersion compensation methods are introduced. In Section 3, we present and discuss the obtained results. Finally, in Section 4, conclusions are drawn.

\section{System setup and parameters}

The setup of the PDM-QPSK coherent optical system is depicted in Fig. 1. The numerical analyses are simulated by OPTISYSTEM 16 and MATLAB platforms.

On the transmitter side, the data is produced by the Pseudo-Random Bit Sequence generator (PRBS-12) of Nbit length. Then, the series of bits are mapped into symbols and converted to pulse forms. The laser Continuous Wave (CW) power is split into two carriers by a Polarization Beam Splitter (PBS). Each of the two parallel I-Q modulators receives half of the laser power. These modulators transfer the four 28 Gbaud electrical signals into two orthogonal 0.3RZ-QPSK signals.

For the proposed system, the pulse used is defined in [19] as:

$$
\begin{aligned}
& g(t)=\operatorname{rect}(2 t / T)-(1 / 2) \operatorname{rect}\left[4\left(t-\frac{3 T}{8}\right) / T\right] \\
& +(1 / 2) \operatorname{rect}\left[4\left(t-\frac{3 T}{8}\right) / T\right]
\end{aligned}
$$

where rect $(t / T)$ is the rectangular pulse of duration $T$. The bandwidth of the proposed pulse is smaller compared to the rectangular pulse and also displays smoother phase changes. The frequency response of the pulse is given by the Fourier transform of Eq. (1) as the following form [17]:

$$
\begin{aligned}
& G(f)=(T / 2) \sin c(f T / 2) \\
& -(T / 4) \cos (3 \pi f T / 4) \sin c(f T / 4) .
\end{aligned}
$$

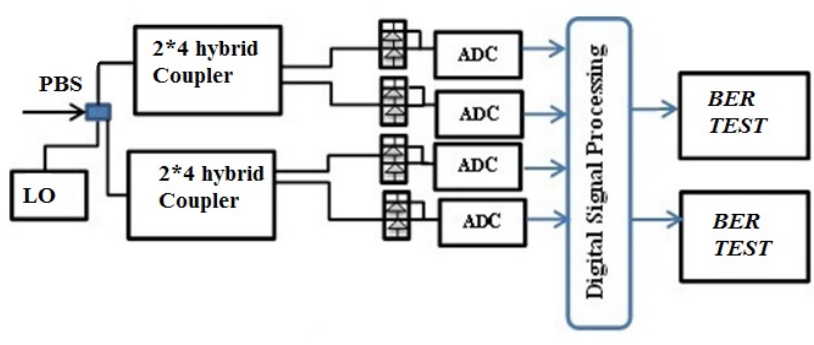

(b)

Fig. 1 Simulation model of PDM-QPSK systems (a) Optical Transmitter (b) Coherent Optical Receiver 
Then, these two signals are multiplexed together with Polarization Beam Combiner (PBC) to form the 0.3RZ-PDM-QPSK optical signal, which propagates into the channel. Table 1 shows the simulation parameters.

The transmission line is composed of spans of SSMF and Erbium-Doped Fiber Amplifiers (EDFAs). EDFAs were used to eliminate the fiber attenuation with $15 \mathrm{~dB}$ and $5 \mathrm{~dB}$ as gain and Noise figure [19]. After that, we have inserted the optical filter with a bandwidth of $\beta_{0}=2 \times R_{s y m}$ to cancel the noise. The optical link characteristics are summarized in Table 2, the Polarization Mode Dispersion (PMD), PN and the nonlinear effect SPM are also presented.

At the receiver end, both of the received optical signals and CW of the Local Oscillator laser (LO) are divided and mixed coherently into two orthogonally polarized branches by PBS. Then, the signals are detected and converted by the photodiodes (PDs) to generate four electrical signals. These signals are processed by a low-pass filter ( $5^{\text {th }}$ order Bessel filter) with a $3 \mathrm{~dB}$ bandwidth of 0.75 times $R_{s y m}$ for interference suppression.

In addition, the Analog-to-Digital Converters (ADCs) synchronously sample the incoming signals by two samples per symbol. The sampling rate is twice of $R_{s y m}$. The digitized signals are treated using the DSP units [20] to mitigate the impairments and recover the data.

The Electronic Dispersion Compensation is implemented using a Frequency Domain Equalizer. Whereas; 5 taps Constant-Modulus Algorithm (CMA) equalizer is

Table 1 Set of transmitter simulation parameters

\begin{tabular}{lc}
\hline Format & PDM-QPSK \\
\hline$R_{b i t}$ & $112 \mathrm{Gbps}$ \\
$R_{\text {sym }}$ & $28 \mathrm{Gbaud}$ \\
$N_{b i t}$ & $2^{12}$ \\
Samples / bit & 64 \\
Number of samples & 262114 \\
Wavelength $(\lambda)$ & $1550 \mathrm{~nm}$ \\
\hline
\end{tabular}

Table 2 The parameters of the optical link at $\lambda=1550 \mathrm{~nm}$

\begin{tabular}{lcc}
\hline Parameters & SSMF & DCF \\
\hline Span length $(L)$ & 75 & LDCF \\
Attenuation $(\alpha)$ & $0.22 \mathrm{~dB} / \mathrm{km}$ & $0.5 \mathrm{~dB} / \mathrm{km}$ \\
Dispersion $(D)$ & $17 \mathrm{ps} / \mathrm{nm} / \mathrm{km}$ & $-85 \mathrm{ps} / \mathrm{nm} / \mathrm{km}$ \\
Dispersion slope $(S)$ & $0.08 \mathrm{ps} / \mathrm{nm}^{2} / \mathrm{km}$ & $-0.21 \mathrm{ps} / \mathrm{nm}^{2} / \mathrm{km}$ \\
Wavelength $(\lambda)$ & $1550 \mathrm{~nm}$ & $1550 \mathrm{~nm}$ \\
Nonlinear refractive index & $2.21 \times 10^{-20} \mathrm{~m}^{2} / \mathrm{W}$ & $2.8 \times 10^{-20} \mathrm{~m}^{2} / \mathrm{W}$ \\
$\left(n_{2}\right)$ & 1.31 & 4.27 \\
Nonlinear coefficient $(\gamma)$ & $80 \mu \mathrm{m}^{2}$ & $22 \mu \mathrm{m}^{2}$ \\
Core effective area $\left(A_{\text {eff }}\right)$ & & \\
\hline
\end{tabular}

realized as a solution for polarization De-multiplexing [21]. As the final step, the carrier phase estimation is performed using a Viterbi-Viterbi algorithm [22] followed by a decision and BER evaluation. In all cases, the Monte Carlo (MC) method [23] is used to estimate the system performance in terms of BER after a noise loading process with at least 400-bit errors. The Bit Error Rate is counted and converted into quality factor (Q-factor) by Eq. (3):

$Q=20 \log _{10}\left[\sqrt{2} \operatorname{erfc}^{-1}(2 \times \mathrm{BER})\right]$

where "erfc" is the error function.

In Subsection 2.1, we will present a brief description of the optical and electronic compensation technique.

\subsection{Optical Dispersion Compensation (ODC)}

The temporal pulse spreading caused by $\mathrm{CD}$ is given by [5]:

$$
\Delta T=D \times z \times \frac{c}{f_{c}^{2}} \times \Delta f
$$

where $D$ is the fiber dispersion parameter, $z$ is the fiber length, $c$ is the speed of light, $f_{c}$ is the carrier frequency, and $\Delta f$ is the pulse spectral width in $\mathrm{Hz}$.

For optical compensation, each span of Single Mode Fiber (SMF) is followed by a DCF with $D$ equal to $-100 \mathrm{ps} / \mathrm{nm} / \mathrm{km}$. The length of the DCF for perfect compensation (dispersion inline $=0$ ) is given by $[24,25]$ :

$$
L_{\mathrm{DCF}}=\left(-\left(L_{\mathrm{SMF}} \times D_{\mathrm{SMF}}\right) / D_{\mathrm{DCF}}\right) \text {. }
$$

\subsection{Electronic Dispersion Compensation (EDC)}

The Overlap-add fast Fourier transform is suggested as a method to mitigate the intersymbol-interference due to Chromatic Dispersion. It is performed by frequency domain $\mathrm{CD}$ equalizer at the receiver side. The incoming signal is divided into small blocks and affixed with zeros at one side. The zero-padded sequence is converted into the frequency domain by Fast-Fourier-Transform (FFT) [26, 27]. Then, the resulting signal is multiplied by the inverse transfer function of the dispersive channel and finally transformed back to the time domain by an Inverse Fast Fourier Transform (IFFT) [18]:

$$
H_{\mathrm{FDE}}=\exp \left(-j \frac{D \lambda^{2} L}{4 \pi c} \omega^{2}\right)
$$

where $\omega, \lambda, c, L$ and $D$ are the angular frequency, wavelength, the speed of light, fiber length and fiber dispersion coefficient, respectively. 
The required zero-padding value is expressed by Eq. (7) [27]:

$N_{\mathrm{FDE}}^{\text {zero-padding }}=2 \times\left\lceil\frac{\sqrt{\pi^{2} c^{2} T^{4}+4 \lambda^{4} D^{2} L^{2}}}{\pi c T^{2}}+1\right\rceil$.

$T=R_{\text {sym }} / 2$ is the sampling period. The FFT-size is twice of the required zero-padding value.

\section{Simulation results and discussion}

In Section 3, the results of the numerical simulations are presented and discussed in order to track the successive transformations of the signal during its propagation.

Fig. 2 shows the time evolution in the phase of the PDM-QPSK optical signal. The four-phase levels of the optical signal $(3 \pi / 4, \pi / 4,-\pi / 4,-3 \pi / 4)$ are observed.

The generated electrical constellation diagrams at each step of DSP algorithms are detailed in Fig. 3. Fig. 3 (a) shows the electrical constellation shape of X-polarization before the DSP algorithm in which the received signal is impacted by the accumulated CD. After applying EDC and the demultiplexing polarization, the constant modulus of the QPSK signal clearly appears in Fig. 3 (b) like a circle. Finally, the step of phase recovery is illustrated in Fig. 3 (c). The data are restored and shaped as four points that signify the four states of phase.

\subsection{Comparison between Optical (ODC) and Electronic Dispersion Compensation (EDC)}

To focus on the mitigation of the Chromatic Dispersion effect, we made a comparison between the Optical and Electronic Dispersion Compensation approaches. The simulations were done in the long-haul single channel 112 Gbps 0.3RZ-PDM-QPSK transmission system.

In these measurements, the transmission is over a fixed distance of $1200 \mathrm{~km}$, corresponding to 16 spans $(75 \mathrm{~km}$ SSMF). While the launch powers are in the range -3 to $2 \mathrm{dBm}$ per channel.

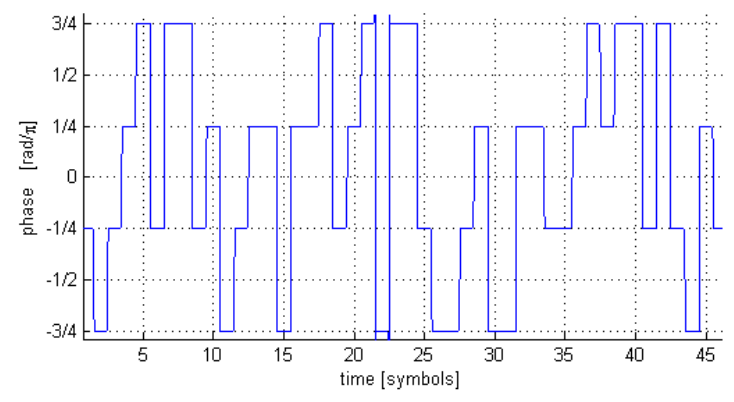

Fig. 2 Temporal representation of the phase of the optical signal
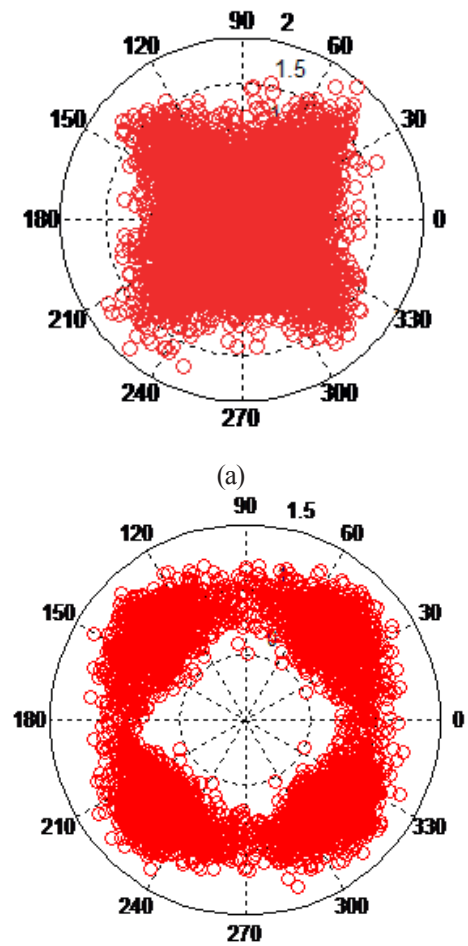

(b)

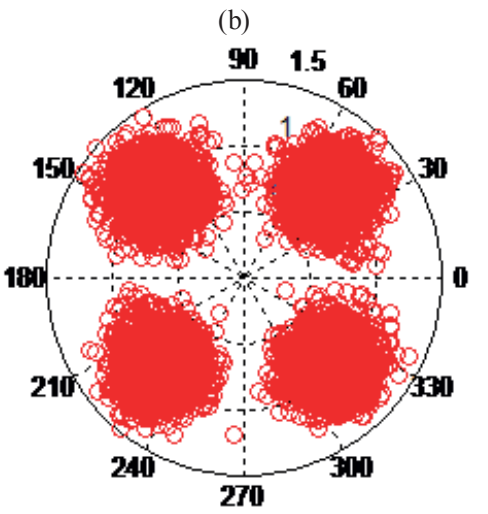

(c)

Fig. 3 Receiver constellation diagrams X-polarization (a) before DSP, (b) after CMA, (c) after Carrier Phase Estimation

The comparison of optical and Electronic Dispersion Compensation is illustrated in Fig. 4 (a) and (b), where the $\mathrm{Q}$ factor and the Bit Error Rate are measured as a function of fiber launch power (Pin) for 112 Gbps 0.3RZ-PDMQPSK system after $1200 \mathrm{~km}$. The DCF is implemented at the end of the optical link, while the FDE is applied in the coherent receiver.

At lower powers, the system performance is limited by ASE noise, then the BER decreases and Q-factor increases until they reach the optimum power. This value is selected at the maximum Q-factor and the minimum BER.

$-1 \mathrm{dBm}$ is considered as optimum power for ODC at BER of $4.9 \times 10^{-4}$ and Q-factor of 10.36. For EDC, the $0 \mathrm{dBm}$ power channel reaches the best performance with 


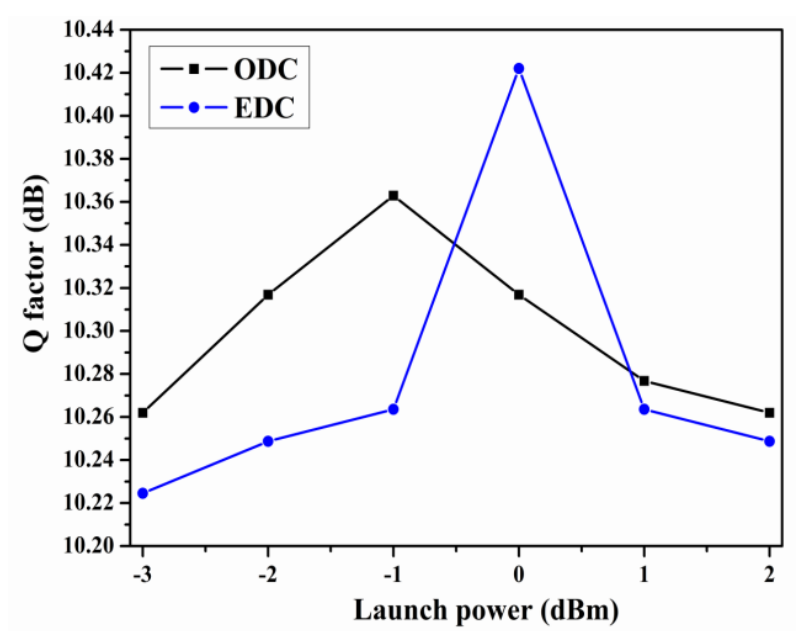

(a)

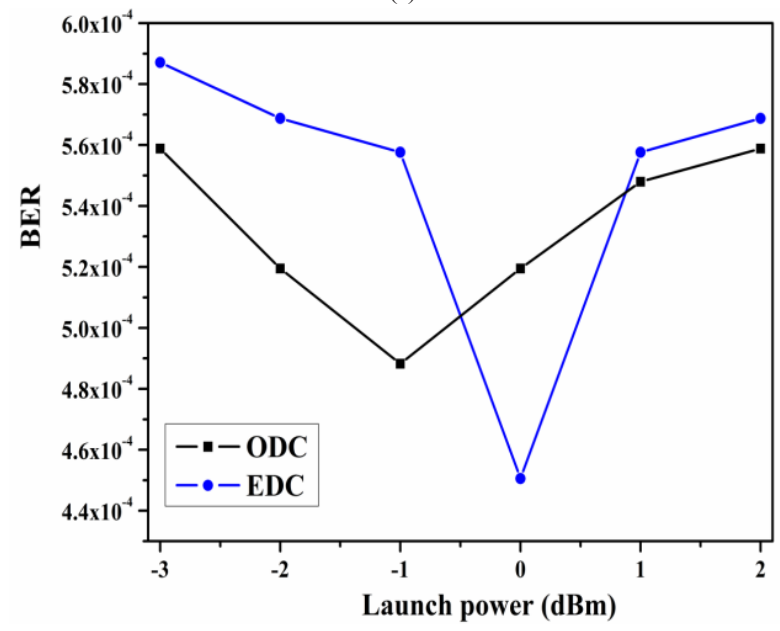

(b)

Fig. 4 (a) Q factor versus launch power for ODC and EDC after $1200 \mathrm{~km}$, (b) BER versus launch power for ODC and EDC after $1200 \mathrm{~km}$

BER of $4.5 \times 10^{-4}$ and Q-factor of approximately $10.44 \mathrm{~dB}$. With the increment in power level, the BER values become important due to the degradation by fiber nonlinearity.

An improvement in the system performance is seen in the case based on FDE compensation. Consequently, Electronic Dispersion Compensation with $0 \mathrm{dBm}$ will be used as the optimum launched power per span for the following measurements.

\subsection{Analysis of the Electronic Dispersion Compensation (EDC)}

In Subsection 3.2, we will investigate the tolerance of Chromatic Dispersion in the two systems. The performances of the 112 Gbps proposed system and 0.3RZ-PDMQPSK system under Back-to-Back (BtB) transmission $(0 \mathrm{~km})$ is studied first. After that, we will evaluate the influence of Chromatic Dispersion on both systems over an uncompensated link.
The total transmission distance is $1200 \mathrm{~km}$, consisting of 16 spans $75 \mathrm{~km}$ SSMF. Also, the OSNR is varied from 12 to $16 \mathrm{~dB}$ using an ASE noise source.

Finally, we will analyze the performance of the two systems after applying the Electronic Dispersion Compensation.

Fig. 5 illustrates both systems in BtB case which is taken as reference. The comparison of two systems plotted in Fig. 6, presented by the $\mathrm{Q}$ factor as a function OSNR $(0.1 \mathrm{~nm})$.

Fig. 6 (a) compares the proposed system without (w/o) and with (w) EDC after $1200 \mathrm{~km}$. In Fig. 6 (b), both systems are using EDC after $1200 \mathrm{~km}$.

Focusing on the Q-factor of the Forward Error Correction (FEC) threshold, the required OSNR for these systems is displayed in Table 3.

It can be seen that both systems show a similar performance between $\mathrm{BtB}$ and Electronic Dispersion Compensation configurations. Also, the OSNR penalty on the 0.3RZ-PDM-QPSK system exceeds that of the proposed system. It is decreasing from $0.33 \mathrm{~dB}$ to $0.14 \mathrm{~dB}$ after using EDC. For the proposed system, the penalties of $0.14 \mathrm{~dB}$ and $0.06 \mathrm{~dB}$ are obtained.

From these results, the proposed system has given a better OSNR tolerance compared to the other system. Furthermore, the ability of the EDC technique at proved in a distance of $1200 \mathrm{~km}$. In Subsection 3.3, the BER performance of the two systems through different fiber lengths is investigated.

\subsection{Influence of fiber transmission distance on EDC}

The performance of both systems is examined in terms of Bit Error Rate at BtB case and different long-haul transmission distances.

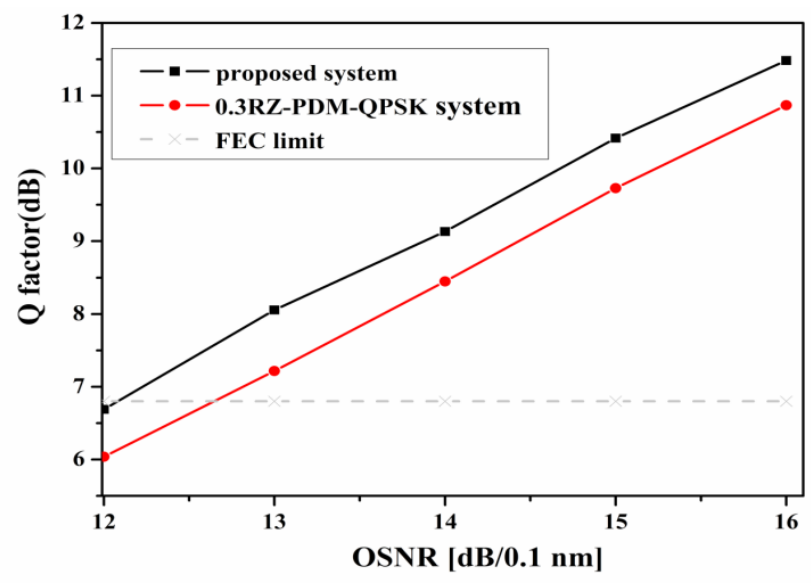

Fig. $5 \mathrm{Q}$ factor as a function of OSNR measured in $0.1 \mathrm{~nm}$ of the two systems in BtB case. 


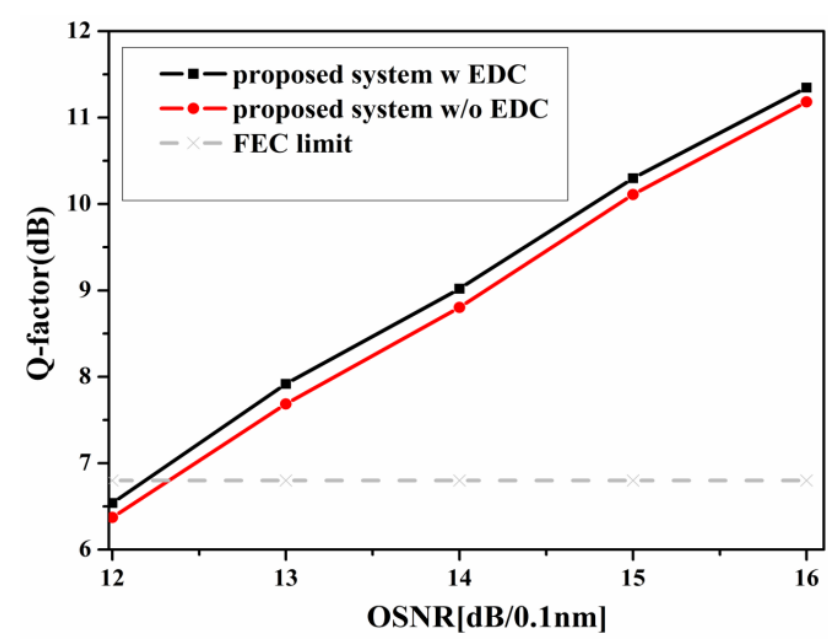

(a)

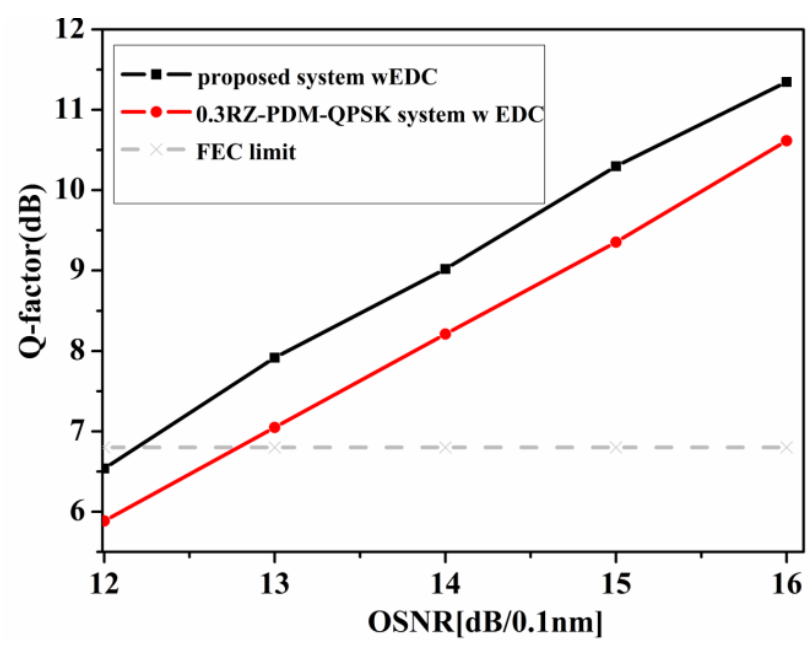

(b)

Fig. $6 \mathrm{Q}$ factor versus OSNR measured in $0.1 \mathrm{~nm}$. (a) for the proposed system without and with compensation, (b) for both systems using EDC

Table 3 The required OSNR at FEC threshold

\begin{tabular}{lcc}
\hline Case & 0.3RZ-PDM-QPSK system & proposed system \\
\hline Back-to-Back & $12.67 \mathrm{~dB}$ & $12.15 \mathrm{~dB}$ \\
w/o EDC & $13 \mathrm{~dB}$ & $12.29 \mathrm{~dB}$ \\
w EDC & $12.81 \mathrm{~dB}$ & $12.21 \mathrm{~dB}$ \\
\hline
\end{tabular}

The BER is generated after the CD compensation for $1200 \mathrm{~km}, 3000 \mathrm{~km}, 4025 \mathrm{~km}$, and $5025 \mathrm{~km}$ fiber lengths. The used spans are 14, 40, 54 and 67, respectively. The Back-to-Back BER was calculated for both systems and plotted here as a reference.

Fig. 7 depicts the measured BER versus OSNR (at $0.1 \mathrm{~nm}$ noise bandwidth) curves of the systems after different transmission lengths.

The required OSNR at FEC limit in back to back transmission is $13.75 \mathrm{~dB}$ and $14 \mathrm{~dB}$ for the proposed system

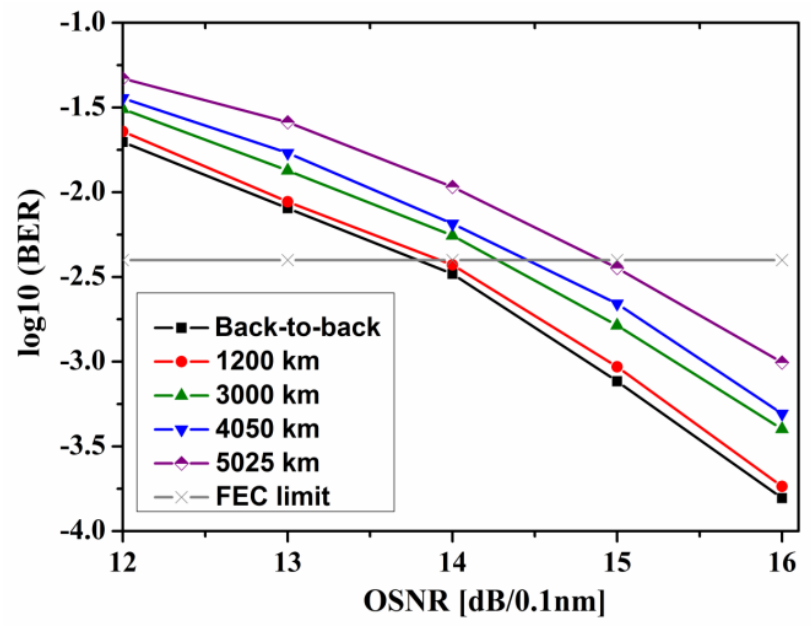

(a) and 0.3RZ-PDM-QPSK system. We calculate the OSNR penalty at BER relative to Back-to-Back for different links and the results are summarized in Table 4, wherein the proposed system is indicated and the 0.3RZ-PDM-QPSK system is referred to.

We can see that the performance of both systems is inversely proportional to the Bit Error Rate and the transmission distance.

In fact, a smaller difference between the performances of both systems at $1200 \mathrm{~km}$ is observed when the penalty is reduced by $0.2 \mathrm{~dB}$. This is due to the robustness of the used pulse with the efficiency of the electronic compensation technique for shorter distances.

Then, the OSNR penalty increases proportionally with the distance. This is clear specifically for the

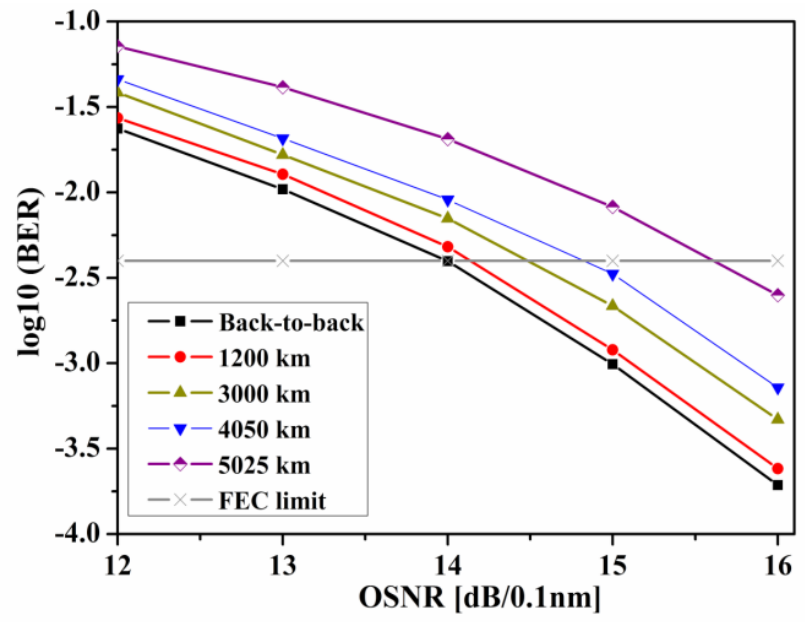

(b)

Fig. 7 Bit Error Rate (BER) as a function of the Optical Signal to Noise Ratio measured in 0.1 nm for the different transmission lengths. (a) proposed system, (b) 0.3RZ-PDM-QPSK system 
Table 4 The OSNR penalty at BER of $3.8 \times 10^{-3}$

\begin{tabular}{lcc}
\hline Distance $(\mathrm{km})$ & OSNR Penalty $^{\mathrm{a}}$ & OSNR Penalty $^{\mathrm{b}}$ \\
\hline 1200 & $0.11 \mathrm{~dB}$ & $0.18 \mathrm{~dB}$ \\
3000 & $0.47 \mathrm{~dB}$ & $0.52 \mathrm{~dB}$ \\
4050 & $0.64 \mathrm{~dB}$ & $0.84 \mathrm{~dB}$ \\
5025 & $1.09 \mathrm{~dB}$ & $1.66 \mathrm{~dB}$ \\
\hline
\end{tabular}

${ }^{a}$ the proposed system, ${ }^{b}$ the $0.3 R Z-P D M-Q P S K$ system

0.3RZ-PDM-QPSK system. It is due to the accumulation of the ASE noise and Chromatic Dispersion with the influence of SPM at long transmission distances. This allows the temporal enlargement of transmitting pulses and increases the intersymbol-interference.

Nevertheless, the proposed system has a higher CD tolerance and a lower penalty compared to the other system.

\subsection{Maximum tolerable transmission distance}

The achievable transmission reach when the performances of the systems are still considerable was calculated after $\mathrm{CD}$ compensation. The measure was done at the optimum optical launch power and fixed OSNR of $16 \mathrm{~dB} / 0.1 \mathrm{~nm}$. The comparison of the measured BER versus the transmission distances is shown in Fig. 8.

According to the results obtained, it can be seen that BER values increased proportionally with higher distances. Both systems achieved a transmission distance up to $5000 \mathrm{~km}$.

The maximum distance which can be reached at BER of $3.8 \times 10^{-3}$ for 112 Gbps 0.3RZ-PDM-QPSK system is $6000 \mathrm{~km}$ with a Q-factor of $9.24 \mathrm{~dB}$. At the same BER, the reach is enhanced to $6412.5 \mathrm{~km}$ with an improvement of $6.88 \%$. The Q-factor attains $9.45 \mathrm{~dB}$.

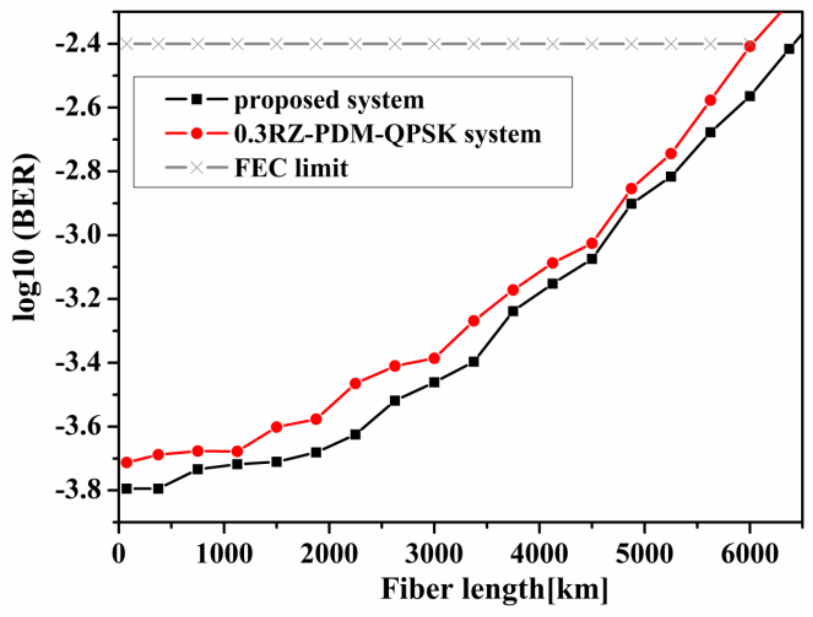

Fig. 8 BER versus transmission distance

\subsection{Influence of symbol-rate on Chromatic Dispersion tolerance}

\subsubsection{Back-to-Back (BtB) characterization}

Initially, the performance of the systems at BtB transmission with different symbol rates was investigated. Both systems were measured with symbol-rates varying from 14 GBaud, 28 GBaud, and 56 Gbaud, respectively.

Fig. 9 shows the Back-to-Back BER as a function of OSNR. Table 5 provides the requirements of Back-toBack OSNRs in both systems at the FEC limit.

However, it is observed that BER degradation has a proportional relationship with a higher symbol rate. In addition, there is an improvement in the performance of the proposed system with better OSNR sensitivity compared to the other system.

\subsubsection{Distance investigation}

The reach of each system at three symbol rates is calculated after applying for electronic compensation. The BER is measured from $75 \mathrm{~km}$ to $8400 \mathrm{~km}$ and plotted in Fig. 10 as a function of distance. The average OSNR is $18 \mathrm{~dB}$. Also, the maximum distance in all cases is displayed in Table 6 .

At higher symbol rates, the degradation of CD tolerance has been observed in simulation results by increasing BER and curtails the maximum reach. The results can be

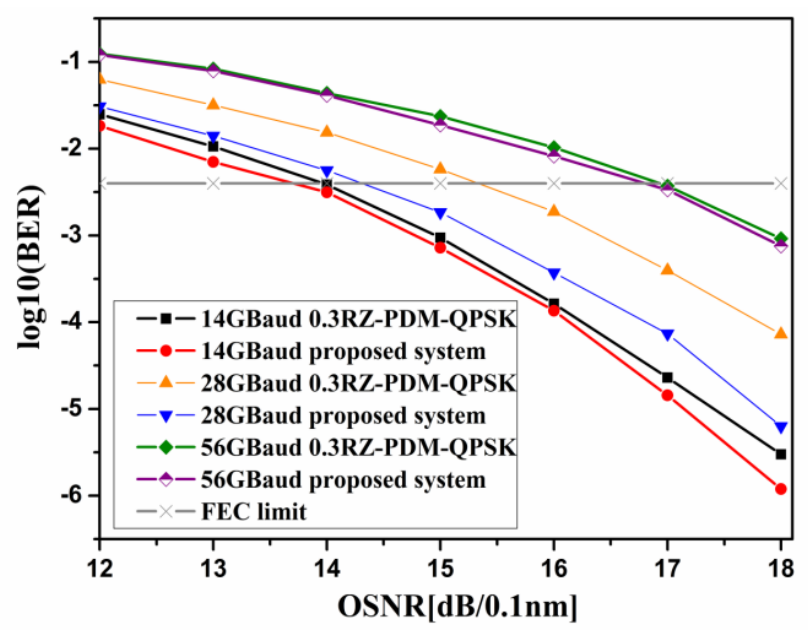

Fig. 9 Back-to-Back measurement at differents symbol rate

Table 5 The Back-to-Back OSNRs at BER of $3.8 \times 10^{-3}$

\begin{tabular}{|c|c|c|}
\hline Symbol-rates & 0.3RZ-PDM-QPSK system & Proposed system \\
\hline 14 Gbaud & $13.91 \mathrm{~dB}$ & $13.67 \mathrm{~dB}$ \\
\hline 28 Gbaud & $15.31 \mathrm{~dB}$ & $14.28 \mathrm{~dB}$ \\
\hline 56 Gbaud & $16.90 \mathrm{~dB}$ & $16.81 \mathrm{~dB}$ \\
\hline
\end{tabular}




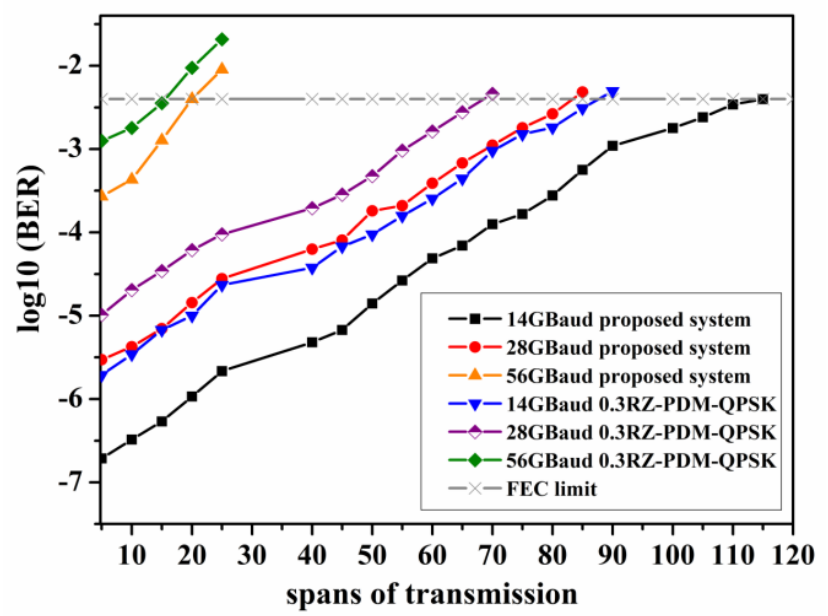

Fig. 10 The BER measured vs. transmission distance

Table 6 The maximum achievable distance at BER of $3.8 \times 10^{-3}$

\begin{tabular}{lcc}
\hline Symbol-rates & 0.3RZ-PDM-QPSK system & Proposed system \\
\hline 14 Gbaud & $6600 \mathrm{~km}$ & $8362.5 \mathrm{~km}$ \\
28 Gbaud & $5212.5 \mathrm{~km}$ & $6262.5 \mathrm{~km}$ \\
56 Gbaud & $1162.5 \mathrm{~km}$ & $1500 \mathrm{~km}$ \\
\hline
\end{tabular}

further improved by using the proposed system. This can also aid in augmenting the number of spans required for the ultra-long coherent optical system.

\section{References}

[1] Taher, K. A., Majumder, S. P. "Analytical Evaluation of the Effect of Cross-Polarization-induced Crosstalk on the BER Performance of a PDM-QPSK Coherent Homodyne Optical Transmission System", Journal of Optical Communications, 38(2), pp. 213-222, 2017. https://doi.org/10.1515/joc-2016-0072

[2] Alvarado, A., Fehenberger, T., Chen, B., Willems, F. M. J. "Achievable Information Rates for Fiber Optics: Applications and Computations", Journal of Lightwave Technology, 36(2), pp. 424-439, 2018. https://doi.org/10.1109/JLT.2017.2786351

[3] Winzer, P. J., Neilson, D. T., Chraplyvy, A. R. "Fiber-optic transmission and networking: the previous 20 and the next 20 years", Optics Express, 26(18), pp. 24190-24239, 2018.

https://doi.org/10.1364/OE.26.024190

[4] Keykhosravi, K., Secondini, M., Durisi, G., Agrell, E. "How to Increase the Achievable Information Rate by Per-Channel Dispersion Compensation", Journal of Lightwave Technology, 37(10), pp. 2443-2451, 2019.

https://doi.org/10.1109/JLT.2019.2907311

[5] Agrawal, G. P. "Fiber-Optic Communication Systems", John Wiley \& Sons Inc., Hoboken, NJ, USA, 2010.

[6] Hussein, T. F., Rizk, M. R. M., Aly, M. H. "A hybrid DCF/FBG scheme for dispersion compensation over a $300 \mathrm{~km} \mathrm{SMF",} \mathrm{Optical}$ and Quantum Electronics, 51(4), Article number: 103, 2019. https://oi.org/10.1007/s11082-019-1823-y

\section{Conclusion}

In this paper, we have demonstrated the robustness of the dispersion compensation in single-channel PDM-QPSK systems at different pulse shapes and symbol rates in terms of Q-factor, BER and OSNR penalty. A comprehensive study of different dispersion compensation techniques was presented. The results indicate that the Frequency Domain Equalizer exhibits the best Q-factor with the optimum launched power of $0 \mathrm{dBm}$ per channel. The proposed system gives a better Chromatic Dispersion tolerance by lowering the OSNR penalty to $0.6 \mathrm{~dB}$ compared to the 0.3RZ-PDM-QPSK system. In addition, it achieves better BER performance with a $6.88 \%$ increment in the reach.

Finally, the expected performances of the proposed system continued even at higher symbol rates and lower OSNR values. The maximum flexible distance will be improved for 14, 28 and 56 Gbaud.

\section{Acknowledgement}

The project presented in this article is supported by the Telecommunications Laboratory, Faculty of Science and Technology, University of May 8, 1945 Guelma, Algeria under project CNEPRU number A25N01UN240120150001.

[7] Ranzini, S. M., Da Ros, F., Bülow, H., Zibar, D. "Tunable Optoelectronic Chromatic Dispersion Compensation Based on Machine Learning for Short-Reach Transmission", Applied Sciences, 9(20), Article number: 4332, 2019.

https://doi.org/10.3390/app9204332

[8] Kudo, R., Kobayashi, T., Ishihara, K., Takatori, Y., Sano, A., Miyamoto, Y. "Coherent Optical Single Carrier Transmission Using Overlap Frequency Domain Equalization for Long-Haul Optical Systems", Journal of Lightwave Technology, 27(16), pp. 3721-3728, 2009.

https://doi.org/10.1109/JLT.2009.2024091

[9] Xu, T., Jacobsen, G., Popov, S., Li, J., Vanin, E., Wang, K., Friberg, A. T., Zhang, Y. "Chromatic dispersion compensation in coherent transmission system using digital filters", Optics Express, 18(15), pp. 16243-16257, 2010. https://doi.org/10.1364/OE.18.016243

[10] Xu, T., Jacobsen, G., Popov, S., Forzati, M., Mårtensson, J., Mussolin, M., Li, J., Wang, K., Zhang, Y., Friberg, A. T. "Frequency-Domain Chromatic Dispersion Equalization Using Overlap-Add Methods In Coherent Optical System", Journal of Optical Communications, 32(2), pp. 131-135, 2011. https://doi.org/10.1515/JOC.2011.022 
[11] Eghbali, A., Johansson, H., Gustafsson, O., Savory, S. J. "Optimal Least-Squares FIR Digital Filters for Compensation of Chromatic Dispersion in Digital Coherent Optical Receivers", Journal of Lightwave Technology, 32(8), pp. 1449-1456, 2014. https://doi.org/10.1109/JLT.2014.2307916

[12] Rozental, V. N., Parahyba, V. E., Reis, J. D., de Oliveira, J. F., Mello, D. A. A. "Digital-domain chromatic dispersion compensation for different pulse shapes: Practical considerations", In: SBMO/IEEE MTT-S International Microwave and Optoelectronics Conference (IMOC), Porto de Galinhas, Brazil, 2015, pp. 1-4. https://doi.org/10.1109/IMOC.2015.7369135

[13] Hraghi, A., Menif, M. "A performance comparison between 33RZ-POMUX-DQPSK and 33RZ-DC-DQPSK using coherent detection for $1.6 \mathrm{~Tb} / \mathrm{s}(16 \times 100 \mathrm{~Gb} / \mathrm{s})$ over $1200 \mathrm{~km}$ ", Proceeding of SPIE 8781, Integrated Optics: Physics and Simulations, Article number: 87811F, 2013.

https://doi.org/10.1117/12.2019469

[14] Alfiad, M. S., Van den Borne, D., Jansen, S. L., Wuth, T., Kuschnerov, M., Grosso, G., Napoli, A., de Waardt, H. "111-Gb/s POLMUX-RZ-DQPSK Transmission over LEAF: Optical versus Electrical Dispersion Compensation", In: $32^{\text {nd }}$ Optical Fiber Communication Conference (OFC), San Diego, USA, 2009, pp. 1-3. https://doi.org/10.1364/OFC.2009.OThR4

[15] Chen, X., Pereda, J. A. M., Horche, P. R. "Signal penalties induced by different types of optical filters in 100 Gbps PM-DQPSK based optical networks", Optical Switching and Networking, 19(3), pp. 145-154, 2016.

https://doi.org/10.1016/j.osn.2015.07.001

[16] Malik, D., Kaushik, G., Wason, A. "Performance Optimization of Optical Amplifiers for High Speed Multilink Optical Networks using Different Modulation Techniques", Journal of Optical Communications, 40(4), pp. 333-340, 2019.

https://doi.org/10.1515/joc-2017-0090

[17] Jain, A., Kumar, K. P. "RF pulse shaping for higher-order optical modulation", In: $20^{\text {th }}$ National Conference on Communications (NCC), Kanpur, India, 2014, pp. 1-4.

https://doi.org/10.1109/NCC.2014.6811250

[18] Xu, T., Jacobsen, G., Popov, S., Li, J., Sergeyev, S., Friberg, A. T., Liu, T., Zhang, Y. "Analysis of chromatic dispersion compensation and carrier phase recovery in long-haul optical transmission system influenced by equalization enhanced phase noise", Optik, 138, pp. 494-508, 2017.

https://doi.org/10.1016/j.ijleo.2017.03.024

[19] Ahlawat, D., Arora, P., Kumar, S. "Performance Evaluation of Proposed WDM Optical Link Using EDFA and FBG Combination", Journal of Optical Communications, 40(2), pp. 101-107, 2019. https://doi.org/10.1515/joc-2018-0044
[20] Li, G., Li, J. "Microwave photonics-based all optical wavelength conversion of Nyquist-DP-16QAM for flex-grid optical networks with 112 Gbps", Microwave and Optical Technology Letters, 61(1), pp. 195-204, 2019.

https://doi.org/10.1002/mop.31543

[21] Nabavi, N. "A full-duplex dual-polarization QPSK in a digital radio-over-fiber system", Microwave and Optical Technology Letters, 60(11), pp. 2698-2704, 2018. https://doi.org/10.1002/mop.31471

[22] Li, Y., Wu, M. W., Du, X., Song, T., Kam, P. Y. "A Refinement to the Viterbi-Viterbi Carrier Phase Estimator and an Extension to the Case With a Wiener Carrier Phase Process", IEEE Access, 7, pp. 78170-78184, 2019.

https://doi.org/10.1109/ACCESS.2019.2922313

[23] Simon, G., Hantos, G. B., Andrade, M. A. B., Desmulliez, M. P. Y., Riehle, M. O., Bernassau, A. L. "Monte-Carlo Based Sensitivity Analysis of Acoustic Sorting Methods", Periodica Polytechnica Electrical Engineering and Computer Science, 63(2), pp. 68-76, 2019. https://doi.org/10.3311/PPee.13455

[24] Ranathive, S., Kumar, K. V., Rashed, A. N. Z., Tabbour, M. S. F., Sundararajan, T. V. P. "Performance Signature of Optical Fiber Communications Dispersion Compensation Techniques for the Control of Dispersion Management", Journal of Optical Communications, 40(1), pp. 93-99, 2019. https://doi.org/10.1515/joc-2019-0021

[25] Giacoumidis, E., Perentos, A., Aldaya, I. "Performance improvement of cascaded dispersion compensation based fiber Bragg gratings by smart selection", Microwave and Optical Technology Letters, 58(12), pp. 2954-2957, 2016. https://doi.org/10.1002/mop.30196

[26] Kakkar, A., Navarro, J. R., Schatz, R., Louchet, H., Pang, X., Ozolins, O., Jacobsen, G., Popov, S. "Comprehensive Study of Equalization-Enhanced Phase Noise in Coherent Optical Systems", Journal of Lightwave Technology, 33(23), pp. 4834-4841, 2015. https://doi.org/10.1109/JLT.2015.2491363

[27] Maanani, Y., Menacer, A. "Modeling and Diagnosis of the Inter-Turn Short Circuit Fault for the Sensorless Input-Output Linearization Control of the PMSM", Periodica Polytechnica Electrical Engineering and Computer Science, 63(3), pp. 159-168, 2019. https://doi.org/10.3311/PPee.13658 